Publ. RIMS, Kyoto Univ.

23 (1987), 895-908

\title{
Non-translation Invariant Product States of Classical Lattice Systems
}

\author{
By \\ Mark FAnNES*
}

\begin{abstract}
The notion of symmetric states is extended in such a way as to include the description of non-translation invariant mean field systems. A complete characterization of such states is given by means of non-translation invariant product states.
\end{abstract}

\section{$\S \mathbb{1}$. Introduction}

A wellknown theorem $[1,2]$ characterizes the symmetric measures on an infinite product of copies of a compact Hausdorff space $\mathcal{K}$, where a measure is called symmetric if it is permutation invariant.

The theorem states that every symmetric measure can be written in a unique way as a convex combination of (symmetric) product measures. This theorem means that a symmetric measure, or equivalently all the correlation functions corresponding to it, can be described with few parameters, namely a measure on the measures on $\mathcal{K}$. This situation generalizes in an natural way to the non-commutative case: it has been shown by St $\varnothing$ rmer [3] and Hudson and Moody [4] that the same theorem holds for symmetric states on an infinite tensor product of $C^{*}$-algebras.

Symmetric states occur in statistical mechanics as equilibrium states of permutation invariant mean field models on a lattice (e.g. the Ising Weiss model, the strongly coupled B.C.S. model, the Dicke maser model, $\cdots[5,6])$. The decomposition theorem for such states together with correlation ine-

Communicated by H. Araki, June 15, 1987.

* Instituut voor Theoretische Fysica, Universiteit Leuven. B-3030 Leuven, Belgium. Bevoegdverklaard Navorser N. F. W. O., Belgium. 
qualities [7] are in fact the main tools which allow to prove the existence and uniqueness of the limiting Gibbs state and to reduce the problem to the study of the gap equation [8].

There are, however, interesting mean field models (such as the discrete B. C. S. model, the continuous B. C. S. model, the H.Y.L. model [9], ‥) for which rigorous proofs of the validity of the gap equation method of computing the equilibrium state don't exist yet $[10,11]$.

A common feature of these models is that their hamiltonians split as a sum of a permutation invariant mean field part and a free part which transforms in a simple way under permutations. The aim of this paper is to provide, at least at the level of classical lattice systems, a decomposition theorem for a class of states which transform under permutations as equilibrium states of the mean field models just mentioned. Such states can of course also be used to give a simple description of systems with temperature gradients or with random fields.

\section{§2. Preliminaries}

In this paper only classical lattice systems are considered. As the geometry of the lattice is irrelevant for our results the points of the lattice are simply enumerated by $N$. To each site $i \in N$ a particle with configuration space $\mathcal{K}$ is attached, where $\mathcal{K}$ is a fixed compact Hausdorff space.

Let $\Lambda \subset N$ be a finite set then $\mathcal{K}^{\Lambda}$ is the configuration space of the subset $\Lambda$ and $\mathcal{A}_{\Lambda}=\mathcal{C}\left(\mathcal{K}^{\Lambda}\right)$, the continuous $\boldsymbol{R}$-valued functions on $\mathcal{K}^{\Lambda}$, are the observables of the region $\Lambda$. Clearly, for $\Lambda_{1} \subset \Lambda_{2} \subset N$ there is a natural embedding of $\mathscr{A}_{\Lambda_{1}}$ in $\mathscr{A}_{\Lambda_{2}}$ which allows to consider $\mathcal{A}_{N}=\mathcal{C}\left(\mathcal{K}^{N}\right)$ as algebra of quasi-local observables as it is the uniform closure of $\bigcup_{\Lambda \in N} \mathcal{A}_{\Lambda}$.

$\mathscr{P}$ will denote the group of local permutations of elements of $N$. Clearly, any $p \in \mathscr{D}$ induces by transposition an automorphism of $\mathcal{A}_{N}$ again denoted by $p$ :

$$
\begin{gathered}
p(f)(x)=f(\tilde{p}(x)) \text { and } \tilde{p}(x)(i)=x(p(i)), \\
f \in \mathcal{A}_{N}, \quad x \in \mathcal{K}^{N}, \quad i \in N .
\end{gathered}
$$

Consider now a 1-body potential $\phi: \mathbb{N} \rightarrow \mathcal{A}_{N}: i \rightarrow \phi_{i} \in \mathcal{A}_{\{i\}}$. The aim of the paper is to characterize the states $\mu$ of $\mathscr{A}_{N}$ (the probability measures on $\mathcal{K}^{N}$ ) which satisfy the following symmetry requirement : 


$$
\mu\left(p^{-1}(f)\right)=\mu(f \exp (-\Delta \phi(p))) \quad p \in \mathscr{Q}, \quad f \in \mathcal{A}_{N}
$$

where

$$
\Delta \phi(p)=\sum_{i}\left(p\left(\phi_{i}\right)-\phi_{i}\right) .
$$

A state $\mu$ satisfying (1) will be called $\phi$-symmetric. Remark that one recovers the usual notion of symmetric state when $\Delta \phi=0$. Equation (1) would also precisely be the D.L.R. equation [12] for trivial local hamiltonians $H_{\Lambda}=\Sigma_{i \in \Lambda} \phi_{i}$ if one would consider a larger class of transformations on $\mathfrak{A}_{N}$ than the local permutations. Indeed, such permutations don't yield information about the behaviour of the state on perturbing $\mathcal{K}$ (the internal degrees of freedom of the particles).

As the potential $\phi$ is not translation-invariant one should impose some growth condition on $\phi$ in order to avoid phase transitions which are due to the non-translation invariance [13]. We will therefore assume that $\phi$ is uniformly bounded :

$$
\sup _{i}\left\|\phi_{i}\right\|<\infty .
$$

\section{§ 3. $\quad \phi$-Symmetric Product States}

We first determine the $\phi$-symmetric product states and we show that they are two by two disjoint if the condition (3) is satisfied.

Proposition III.1. Let $\times_{i \in N} \mu_{2}$ be a $\phi$-symmetric (product) state of $\mathcal{A}_{N}$, where each of the $\mu_{i}$ is a probability measure on $\mathcal{K}$, then there exists a probability measure $\mu$ on $\mathcal{K}$ such that

$$
\mu_{i}(d x)=\frac{\mu(d x) \exp \left(-\phi_{i}(x)\right)}{\mu\left(\exp \left(-\phi_{i}\right)\right)} \quad x \in \mathcal{K}, \quad i \in \mathbb{N} .
$$

Proof. Choose $i \in\{2,3, \cdots\}$ and let $p$ be the permutation

$$
\left(\begin{array}{cc}
1 & i \\
i & 1
\end{array}\right)
$$

For $f \in \mathcal{A}_{\{1\}}$ it then follows from the $\phi$-symmetry of $\times_{i \in N} \mu_{i}$ that

$$
\mu_{i}(f)=\underset{j \in N}{\times} \mu_{j}\left(p^{-1}(f)\right)
$$




$$
\begin{aligned}
& =\underset{j \in N}{\times} \mu_{j}(f \exp (-\Delta \phi(p))) \\
& =\mu_{1}\left(f \exp \left(\phi_{1}-\phi_{\imath}\right)\right) \mu_{2}\left(\exp \left(\phi_{i}-\phi_{1}\right)\right) .
\end{aligned}
$$

By normalisation

$$
1=\mu_{1}\left(\exp \left(\phi_{1}-\phi_{i}\right)\right) \mu_{i}\left(\exp \left(\phi_{i}-\phi_{1}\right)\right) .
$$

It is now sufficient to put

$$
\mu(d x)=\frac{\mu_{1}(d x) \exp \left(\phi_{1}(x)\right)}{\mu_{1}\left(\exp \left(\phi_{1}\right)\right)}, \quad x \in \mathcal{K}
$$

to get (4).

We now recall a theorem of Kakutani that characterizes absolute continuity for product measures.

Theorem II. 2 [14]。 Let $\times_{i \in N} \mu_{i}$ and $\times_{i \in N} \nu_{2}$ be product probability measures on $\mathcal{K}^{N}$ such that for $i \in \mathbb{N}, \mu_{i}$ is absolutely continuous w.r.t $\nu_{i}$ : $\exists$ a non-negative $\nu_{i}$ measurable function $f_{i}$ such that

$$
\mu_{i}(d x)=\nu_{i}(d x) f_{i}(x), \quad x \in \mathcal{K}, \quad i \in N .
$$

Then $\underset{i \in N}{\times} \mu_{i}$ is absolutely continous, respectively disjoint, w.r.t. $\underset{i \in N}{\times} \nu_{i}$ accord . ing to whether

$$
\sum_{i \in N}\left(1-\nu_{i}\left(f_{i}^{1 / 2}\right)\right)
$$

is convergent or divergent.

Lemma III. 3. Let $\lambda$ be a probability measure on a measure space $\Omega$, $\omega \rightarrow f(\omega) \in \mathbb{R}^{+}$a measurable function on $\Omega$ and $\Omega_{1} \subset \Omega$ a measurable subset of $\Omega$ then:

$$
\frac{\int_{\Omega} \lambda(d \omega) f^{1 / 2}(\omega)}{\left(\int_{\Omega} \lambda(d \omega) f(\omega)\right)^{1 / 2}} \leq\left(\frac{\left(\int_{\Omega_{1}} \lambda(d \omega) f^{1 / 2}(\omega)\right)^{2}}{\int_{\Omega_{1}} \lambda(d \omega) f(\omega)}+1-\int_{\Omega_{1}} \lambda(d \omega)\right)^{1 / 2}
$$

Proof. One has by the Schwartz inequality:

$$
\begin{aligned}
\int_{\Omega} \lambda(d \omega) f^{1 / 2}(\omega)= & \int_{\Omega_{1}} \lambda(d \omega) f^{1 / 2}(\omega)+\int \lambda(d \omega) f^{1 / 2}(\omega) \\
& \leq \int_{\Omega_{1}} \lambda(d \omega) f^{1 / 2}(\omega)+\left(\int_{\Omega_{\backslash} \Omega_{1}} \lambda(d \omega)\right)^{1 / 2}\left(\int_{\Omega_{\curlywedge} \Omega_{1}} \lambda(d \omega) f(\omega)\right)^{1 / 2}
\end{aligned}
$$




$$
=\int_{\Omega_{1}} \lambda(d \omega) f^{1 / 2}(\omega)+\left(1-\int_{\Omega_{1}} \lambda(d \omega)\right)^{1 / 2}\left(\int_{\Omega_{\backslash} \Omega_{1}} \lambda(d \omega) f(\omega)\right)^{1 / 2}
$$

Therefore

$$
\frac{\int_{\Omega} \lambda(d \omega) f^{1 / 2}(\omega)}{\left(\int_{\Omega} \lambda(d \omega) f(\omega)\right)^{1 / 2}} \leq \frac{\int_{\Omega_{1}} \lambda(d \omega) f^{1 / 2}(\omega)+\left(1-\int_{\Omega_{1}} \lambda(d \omega)\right)^{1 / 2}\left(\int_{\Omega \backslash \Omega_{1}} \lambda(d \omega) f(\omega)\right)^{1 / 2}}{\left(\int_{\Omega_{1}} \lambda(d \omega) f(\omega)+\int_{\Omega_{\Omega} \Omega_{1}} \lambda(d \omega) f(\omega)\right)^{1 / 2}}
$$

Let now $a, b$ and $c$ belong to $\mathbb{R}^{+}$then the function

$$
x \in \mathbb{R}^{+} \rightarrow \frac{a+b x^{1 / 2}}{(c+x)^{1 / 2}} \in \mathbb{R}^{+}
$$

reaches its supremum at $x=\left(b^{2} c^{2} / a^{2}\right)$ where it attains the value $\left(a^{2} / c\right.$ $\left.+b^{2}\right)^{1 / 2}$. Putting now

$$
\begin{aligned}
& a=\int_{\Omega_{1}} \lambda(d \omega) f^{1 / 2}(\omega) \\
& b=\left(1-\int_{\Omega_{1}} \lambda(d \omega)\right)^{1 / 2} \\
& c=\int_{\Omega_{1}} \lambda(d \omega) f(\omega)
\end{aligned}
$$

the lemma follows.

Proposition $\mathbb{1}$ I. 4. The $\phi$-symmetric product states are two by two disjoint if

$$
\gamma=\sup _{i}\left\|\phi_{i}\right\|<\infty
$$

Proof. Let $\nu$ be a probability measure on $\mathcal{K}$ and choose a nonnegative $\nu$-measurable function $f$ such that $\nu(f)=1$. The measures $\mu$ and $\nu$, where $\mu(d x)=\nu(d x) f(x), x \in \mathcal{K}$, will be unequal iff there exist measurable sets $\mathcal{O}_{1}, \mathcal{O}_{2} \subset \mathcal{K}$ such that

$$
\underset{y_{1} \in \mathcal{O}}{\operatorname{ess}} \inf _{y_{2} \in \mathcal{O}_{2}}\left|f\left(y_{1}\right)-f\left(y_{2}\right)\right| \nu\left(\mathcal{O}_{1}\right) \nu\left(\mathcal{O}_{2}\right)>0
$$

By Proposition III. 1 and Theorem III. 2 we have to show that

$$
\sum_{i \in N}\left(1-\frac{\nu\left(f^{1 / 2} \exp \left(-\phi_{i}\right)\right)}{\left(\nu\left(f \exp \left(-\phi_{i}\right)\right)\right)^{1 / 2}\left(\nu\left(\exp \left(-\phi_{2}\right)\right)\right)^{1 / 2}}\right)=+\infty
$$

if (7) is satisfied. 
We now apply for each $i \in N$ Lemma III. 3 with $\Omega=\mathcal{K}, \Omega_{1}=\mathcal{O}_{1} \cup \mathcal{O}_{2}$ and

$$
\lambda(d x)=\frac{\nu(d x) \exp \left(-\phi_{i}(x)\right)}{\nu\left(\exp \left(-\phi_{i}\right)\right)}
$$

Denoting by $\chi_{\mho}, \mathcal{U} \subset \mathcal{K}$, the characteristic function of $\mathcal{U}$ one finds :

$$
\begin{aligned}
& 1-\frac{\nu\left(f^{1 / 2} \exp \left(-\phi_{i}\right)\right)}{\left(\nu\left(f \exp \left(-\phi_{i}\right)\right)\right)^{1 / 2}\left(\nu\left(\exp \left(-\phi_{i}\right)\right)\right)^{1 / 2}} \\
& \geq 1-\left(1-\left(\frac{\nu\left(\chi \sigma_{1} \cup \theta_{2} \exp \left(-\phi_{i}\right)\right)}{\nu\left(\exp \left(-\phi_{i}\right)\right)}\right.\right. \\
& \left.\left.-\frac{\nu\left(\chi O_{1} \cup \mathcal{O}_{2} f^{1 / 2} \exp \left(-\phi_{i}\right)\right)^{2}}{\nu\left(\exp \left(-\phi_{i}\right)\right) \nu\left(\chi \Theta_{1} \cup \mathcal{O}_{2} f \exp \left(-\phi_{i}\right)\right)}\right)\right)^{1 / 2} \\
& \geq \frac{1}{2} \frac{\nu\left(\chi O_{1} \cup O_{2} \exp \left(-\phi_{i}\right)\right) \nu\left(\chi O_{1} \cup O_{2} f \exp \left(-\phi_{i}\right)\right)-\nu\left(\chi O_{1} \cup O_{2} Z^{1 / 2} \exp \left(-\phi_{i}\right)\right)^{2}}{\nu\left(\exp \left(-\phi_{i}\right)\right) \nu\left(\chi O_{1} \cup O_{2} f \exp \left(-\phi_{i}\right)\right)} \\
& \left(\text { for } x \leq 1: 1-(1-x)^{1 / 2} \geq \frac{1}{2} x\right) \\
& =\frac{1}{4} \frac{\left.\int \nu\left(d y_{1}\right) \nu\left(d y_{2}\right) f^{1 / 2}\left(y_{1}\right)-f^{1 / 2}\left(y_{2}\right)\right)^{2} \chi \theta^{1} \cup \theta^{2}\left(y_{1}\right) \chi \sigma^{1} \cup \theta^{2}\left(y_{2}\right) \exp \left(-\phi_{i}\left(y_{1}\right)-\phi_{i}\left(y_{2}\right)\right)}{\nu\left(\exp \left(-\phi_{i}\right)\right) \nu\left(\chi \sigma^{1} \cup \theta^{2} f \exp \left(-\phi_{i}\right)\right)} \\
& \geq \frac{1}{2} \frac{\underset{y_{1} \in \mathcal{O}_{1} y_{2} \in \mathcal{O}_{2}}{\operatorname{ess}} \inf _{f_{1}}\left(f^{1 / 2}\left(y_{1}\right)-f^{1 / 2}\left(y_{2}\right)\right)^{2} \nu\left(\mathcal{O}_{1}\right) \nu\left(\mathcal{O}_{2}\right) \exp (-2 \gamma)}{\nu\left(\exp \left(-\phi_{i}\right)\right) \nu\left(\chi \mathcal{O}_{1} \cup \mathcal{O}_{2} f \exp \left(-\phi_{i}\right)\right)} \\
& \geq \delta>0
\end{aligned}
$$

where by (7) and the uniform boundedness of $\phi \delta$ can be chosen independent of $i$. Therefore (8) is satisfied.

\section{§ 4. Decomposition of $\phi$-Symmetric States}

Lemma IV. 1. Let $a=\left(a_{i}\right)_{i=1, \cdots, N}$ and $b=\left(b_{i}\right)_{i=1, \cdots, N}$ belong to $\left(\boldsymbol{R}^{+}\right)^{N}$, $N=1,2, \cdots$

$$
A_{i j}=a_{i} b_{j} \quad i, j=1, \cdots, N
$$

and

$$
\gamma=\|a\|\|b\|-\langle a \mid b\rangle
$$

then

$$
\left[A_{i j}\right]+\frac{1}{2} \gamma\left[\delta_{i, j}\right]
$$


is a positive matrix on $\mathbb{R}^{N}$.

Proof. If $\gamma=0$ and $\left(a_{i}\right)_{i=1, \cdots, N} \neq 0$ then there is a $\lambda \geq 0$ such that $b_{i}=\lambda a_{i}$ $i=1, \cdots, N$. $\left[A_{i j}\right]$, considered as a linear operator on $\mathbb{C}^{N}$, is $\lambda\|a\|^{2}$ times the orthogonal projection on $\mathbb{C}_{a}$ and therefore positive on $\mathbb{C}^{N}$ and hence on $\mathbb{R}^{N}$.

If $\gamma \neq 0$ then $a$ and $b$ are linearly independent in $\mathbb{C}^{N}$ and it is sufficient to show that

$$
a \otimes b+b \otimes a+\gamma \mathbb{1}
$$

is a positive operator on $C^{N}$ where

$$
(\phi \otimes \psi) \chi=\langle\phi \mid \chi\rangle \phi \quad \phi, \phi, \chi \in \mathbb{C}^{N} .
$$

By an explicit computation however it is easy to check that the lowest eigenvalue of the rank 2 operator $a \otimes b+b \otimes a$ is precisely $-\gamma$.

The next lemma is the main result needed to characterize the extreme $\phi$-symmetric states.

Lemma IV.2. Let $\phi$ be a uniformly bounded 1-body potential and let $\mu$ be a $\phi$-symmetric state of $\mathcal{A}_{N}$.

Suppose that

$$
\begin{aligned}
& g \in \mathcal{A}_{\Lambda_{0}}, \quad g \geq 0 \\
& f \in \mathcal{A}_{\Lambda}, \quad \Lambda \cap \Lambda_{0}=\emptyset \\
& p \in \mathscr{Q} \text { is such that } p(\Lambda) \cap \Lambda_{0}=\emptyset \text { and } p(\Lambda) \cap \Lambda=\emptyset
\end{aligned}
$$

then

$$
\mu\left(g f p(f) \exp \left(\sum_{i \in \Lambda} \phi_{i}+\sum_{i \in p(\Lambda)} \phi_{i}\right)\right) \geq 0 .
$$

Proof. Let $\Lambda=\left\{i_{1}, \cdots, i_{r}\right\}$ and $p(\Lambda)=\left\{j_{1}, \cdots, j_{r}\right\}$ where $p\left(i_{k}\right)=j_{k}, k=1$, $\cdots, r$.

Denote by $\mu_{1}$ the measure $\mu$ restricted to $\mathcal{A}_{\{1\}}$. As $\gamma=\sup _{i}\left\|\phi_{i}\right\|<\infty$ we can always choose a strictly increasing sequence $\left\{n_{1}, n_{2}, \cdots\right\}$ in $\mathbb{N} \backslash \Lambda_{0} \cup \Lambda \cup p(\Lambda)$ and $\phi_{\infty} \in \mathcal{L}^{\infty}\left(\mathcal{K}, \mu_{1}\right)$ such that $\left\|\phi_{\infty}\right\| \leq \gamma$ and

$$
\sum_{k} \int_{\mathcal{K}} \mu_{1}(d y)\left(\phi_{n_{k}}(y)-\phi_{\infty}(y)\right)^{2}<\infty .
$$

Let now $\Lambda^{k}=\left\{n_{(k-1) r+1}, n_{(k-1) r+2}, \cdots, n_{k}\right\}, k=1,2, \cdots$ and define local permutations $p_{k}, q_{k} \in \mathscr{Q}$ by 


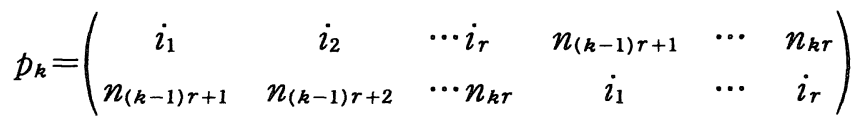

$$
\begin{aligned}
& q_{k}=\left(\begin{array}{cccccc}
j_{1} & j_{2} & \cdots j_{r} & n_{(k-1) r+1} & \cdots & n_{k r} \\
n_{(k-1) r+1} & n_{(k-1) r+2} & \cdots n_{k r} & j_{1} & \cdots & j_{r}
\end{array}\right) .
\end{aligned}
$$

For $M \subset N$ finite we will use the shorthand notation

$$
\phi(M)=\sum_{i \in M} \phi_{i}
$$

By the $\phi$-symmetry of $\mu$ one has for $k \neq l$

$$
\begin{aligned}
\mu(g f p(f) \exp (\phi(\Lambda)+\phi(p(\Lambda)))) \\
=\mu\left(g \exp (\phi(\Lambda)+\phi(p(\Lambda))) p_{k}\left(\exp \left(-\phi\left(\Lambda^{k}\right)\right)\right) \exp \left(\phi\left(\Lambda^{k}\right)\right) p_{k}(f)\right. \\
\left.\quad q_{l}\left(\exp \left(-\phi\left(\Lambda^{l}\right)\right)\right) \exp \left(\phi\left(\Lambda^{l}\right)\right) p_{l}(f)\right)
\end{aligned}
$$

We now apply Lemma III. 1 with the choice

$$
a_{k}=p_{k}\left(\exp \left(-\phi\left(\Lambda^{k}\right)\right)\right) \quad b_{k}=q_{k}\left(\exp \left(-\phi\left(\Lambda^{k}\right)\right)\right) \quad k=1, \cdots, N
$$

to obtain that

(13) $\sum_{k, l=1}^{N} \exp \left(\phi\left(\Lambda^{k}\right)\right) p_{k}(f) p_{k}\left(\exp \left(-\phi\left(\Lambda^{k}\right)\right)\right) q_{l}\left(\exp \left(-\phi\left(\Lambda^{l}\right)\right)\right) \exp \left(\phi\left(\Lambda^{l}\right)\right) p_{l}(f)$

$$
\geq-\frac{1}{2} \gamma(N) \sum_{k=1}^{N} \exp \left(2 \phi\left(\Lambda^{k}\right)\right) p_{k}(f)^{2}
$$

where

$$
\begin{aligned}
\gamma(N)= & \left(\sum_{k=1}^{N} p_{k}\left(\exp \left(-2 \phi\left(\Lambda^{k}\right)\right)\right)\right)^{1 / 2}\left(\sum_{k=1}^{N} q_{k}\left(\exp \left(-2 \phi\left(\Lambda^{k}\right)\right)\right)\right)^{1 / 2} \\
& -\sum_{k=1}^{N} p_{k}\left(\exp \left(-\phi\left(\Lambda^{k}\right)\right)\right) q_{k}\left(\exp \left(-\phi\left(\Lambda^{k}\right)\right)\right)
\end{aligned}
$$

Multiplying both sides of (13) with the positive function $g \exp (\phi(\Lambda)$ $+\phi(p(\Lambda)))$ and taking the expectation with respect to $\mu$ one finds by (12):

$$
\begin{aligned}
& N(N-1) \mu(g f p(f) \exp (\phi(\Lambda)+\phi(p(\Lambda)))) \\
& \quad+\sum_{k=1}^{N} \mu\left(g \exp (\phi(\Lambda)+\phi(p(\Lambda))) \exp \left(2 \phi\left(\Lambda^{k}\right)\right) p_{k}(f)^{2}\right. \\
& \left.\left.\quad \times p_{k}\left(\exp \left(-\phi \Lambda^{k}\right)\right)\right) q_{k}\left(\exp \left(-\phi\left(\Lambda^{k}\right)\right)\right)\right) \\
& \geq-\frac{1}{2} \sum_{k=1}^{N} \mu\left(g \exp (\phi(\Lambda)+\phi(p(\Lambda))) \exp \left(2 \phi\left(\Lambda^{k}\right)\right) p_{k}(f)^{2} \gamma(N)\right) .
\end{aligned}
$$


But

$$
\begin{gathered}
\sum_{k=1}^{N} \mu\left(g \exp (\phi(\Lambda)+\phi(p(\Lambda))) \exp \left(2 \phi\left(\Lambda^{k}\right)\right) p_{k}(f)^{2} p_{k}\left(\exp \left(-\phi\left(\Lambda^{k}\right)\right)\right)\right. \\
\left.q_{k}\left(\exp \left(-\phi\left(\Lambda^{k}\right)\right)\right)\right) \leq N\|g\|\|f\|^{2} \exp (6 \gamma r) \\
\sum_{k=1}^{N} \mu\left(g \exp (\phi(\Lambda)+\phi(p(\Lambda))) \exp \left(2 \phi\left(\Lambda^{k}\right)\right) p_{k}(f)^{2} \gamma(N)\right) \\
\leq N\|g\|\|f\|^{2} \exp (4 \gamma r) \mu(\gamma(N))
\end{gathered}
$$

Now for $x=\left(x_{1}, \cdots, x_{r}\right) \in \mathcal{K}^{\Lambda}$ and $y=\left(y_{1}, \cdots, y_{r}\right) \in \mathcal{K}^{p(\Lambda)}$

$$
\begin{aligned}
0 \leq & \gamma(N)(x, y)=\left(\left(\sum_{k=1}^{N} p_{k}\left(\exp \left(-2 \phi\left(\Lambda^{k}\right)\right)\right)\right)^{1 / 2}\left(\sum_{k=1}^{N} q_{k}\left(\exp \left(-2 \phi\left(\Lambda^{k}\right)\right)\right)\right)^{1 / 2}\right. \\
& \left.-\sum_{k=1}^{N} p_{k}\left(\exp \left(-2 \phi\left(\Lambda^{k}\right)\right)\right) q_{k}\left(\exp \left(-2 \phi\left(\Lambda^{k}\right)\right)\right)\right)(x, y) \\
= & \left(\prod_{i=1}^{r} \exp \left(-\phi_{\infty}\left(x_{i}\right)-\phi_{\infty}\left(y_{i}\right)\right)\right) \\
& \left(\left(\sum_{k=1}^{N} \prod_{i=1}^{r} \exp \left(-2 \phi_{n_{(k-1) r+2}}\left(x_{i}\right)+2 \phi_{\infty}\left(x_{i}\right)\right)\right)^{1 / 2}\right. \\
& \left(\sum_{k=1}^{N} \prod_{i=1}^{r} \exp \left(-2 \phi_{n_{(k-1) r+z}}\left(y_{i}\right)+2 \phi_{\infty}\left(y_{i}\right)\right)\right)^{1 / 2} \\
& \left.-\sum_{k=1}^{N} \prod_{i=1}^{r} \exp \left(-\phi_{n_{(k-1) r+2}}\left(x_{i}\right)+\phi_{\infty}\left(x_{i}\right)-\phi_{n(k-1) r+z}\left(y_{i}\right)+\phi_{\infty}\left(y_{i}\right)\right)\right) \\
\leq & \frac{1}{2} \exp (2 \gamma r) \sum_{k=1}^{N}\left(\prod_{i=1}^{r} \exp \left(-\phi_{n(k-1) r+2}\left(x_{i}\right)+\phi_{\infty}\left(x_{i}\right)\right)\right. \\
& \left.-\prod_{i=1}^{r} \exp \left(-\phi_{n_{(k-1) r+2}}\left(y_{i}\right)+\phi_{\infty}\left(y_{i}\right)\right)\right)^{2} \\
\leq & \exp (2 \gamma r)\left(\sum_{k=1}^{N}\left(\prod_{i=1}^{r} \exp \left(-\phi_{n_{(k-1) r+t}}\left(x_{i}\right)+\phi_{\infty}\left(x_{i}\right)\right)-1\right)^{2}\right. \\
& \left.+\sum_{k=1}^{N}\left(\prod_{i=1}^{r} \exp \left(-\phi_{n_{(k-1) r+i}}\left(y_{i}\right)+\phi_{\infty}\left(y_{i}\right)\right)-1\right)^{2}\right)
\end{aligned}
$$

Using the shorthand notation

$$
a_{i}=\exp \left(-\phi_{n_{(k-1) r+i}}\left(x_{2}\right)+\phi_{\infty}\left(x_{i}\right)\right) \quad i=1, \cdots, r
$$

one has

$$
\left|a_{i}\right| \leq \exp (2 \gamma)
$$

and by convexity of the exponential function

$$
\left|a_{i}^{-1}\right| \leq 1-\phi_{\infty}\left(x_{i}\right)-\phi_{n(k-1) r+i}\left(x_{i}\right) \mid \exp (2 \gamma) .
$$


We then estimate :

$$
\begin{aligned}
& \left(a_{1} \cdots a_{r}-1\right)^{2}= \\
& \quad\left(\left(a_{1}-1\right) a_{2} \cdots a_{r}+\left(a_{2}-1\right) a_{3} \cdots a_{r}+\cdots+\left(a_{r}-1\right)\right)^{2} \leq \\
& \quad r\left(\left(a_{1}-1\right)^{2} a_{2}^{2} \cdots a_{r}^{2}+\left(a_{2}-1\right)^{2} a_{3}^{2} \cdots a_{r}{ }^{2}+\cdots+\left(a_{r}-1\right)^{2}\right) \leq \\
& \quad r \exp (4 \gamma r)\left(\sum_{i=1}^{r}\left(\phi_{\infty}\left(x_{i}\right)-\phi_{n_{(k-1) r+i}}\left(x_{i}\right)\right)^{2}\right) .
\end{aligned}
$$

Using the $\phi$-symmetry of $\mu$ one has

$$
\mu\left(\left(\phi_{\infty}\left(x_{i}\right)-\phi_{n_{(k-1) r+i}}\left(x_{i}\right)\right)^{2}\right) \leq \exp (4 \gamma) \int_{\mathcal{K}} \mu_{1}(d y)\left(\phi_{\infty}(y)-\phi_{n_{(k-1) r+i}}(y)\right)^{2}
$$

where $\mu_{1}$ is the measure $\mu$ restricted to $\mathcal{A}_{\{1\}}$. It finally follows that

$$
\mu(\gamma(N)) \leq 2 r \exp (10 \gamma r) \sum_{k=1}^{N r} \int_{\mathcal{K}} \mu_{1}(d y)\left(\phi_{\infty}(y)-\phi_{n_{k}}(y)\right)^{2}
$$

which is by (11) uniformly bounded in $N$. The lemma now follows by taking the limit $N \rightarrow \infty$ in (15).

Corollary IV. 3. Let $\phi$ be a uniformly bounded 1-body potential and let $\mu$ be a $\phi$-symmetric state of $\mathcal{A}_{N}$. Suppose that there exist two finite subsets $\Lambda_{1}$ and $\Lambda_{2}$ of $N$ such that $\Lambda_{1} \cap \Lambda_{2}=\emptyset$ and $\mu_{\Lambda_{1} \cup \Lambda_{2}}=\mu_{\Lambda_{1}} \times \mu_{\Lambda_{2}}$ where for $\Lambda \subset N \mu_{\Lambda}=\left.\mu\right|_{\AA^{A}}$.

For any two subsets $M_{1}, M_{2}$ of $N$ such that $M_{1} \cap M_{2}=\emptyset$ and $\#\left(M_{i}\right) \leq$ \# $\left(\Lambda_{i}\right) i=1,2$ one has

$$
\mu_{M_{1} \cup M_{2}}=\mu_{M_{1}} \times \mu_{M_{2}} .
$$

Proof. One can without loss of generality assume that $\S\left(\Lambda_{1}\right) \leq\left(\Lambda_{2}\right)$ and that $\#\left(M_{1}\right)=\#\left(\Lambda_{i}\right) i=1,2$.

Consider now any $g \in \mathcal{A}_{M_{1}}$ and $r \in \mathscr{Q}$ such that $r\left(M_{1}\right) \subset M_{2}, r^{2}=$ id and $r=$ id on $N \backslash\left(M_{1} \cup r\left(M_{1}\right)\right)$. By Lemma IV. 2 one has then for all $\lambda \in \boldsymbol{R}$ :

$$
0 \leq \mu\left((g-\lambda)(r(g)-\lambda) \exp \left(\phi\left(M_{1} \cup r\left(M_{1}\right)\right)\right)\right) .
$$

Choose now $q \in \mathscr{Q}$ such $q\left(M_{1}\right)=\Lambda_{1}, q \cdot r\left(M_{1}\right) \subset \Lambda_{2}, q^{2}=\mathrm{id}$ and $q=\mathrm{id}$ on $N \backslash N$ where $N=M_{1} \cup r\left(M_{2}\right) \cup \Lambda_{1} \cup q \cdot r\left(M_{1}\right)$. Defining $p=q \cdot r \cdot q$ one has $p\left(\Lambda_{1}\right)$ $\subset \Lambda_{2}, p^{2}=\mathrm{id}$ and $p=\mathrm{id}$ on $N \backslash\left(\Lambda_{1} \cup p\left(\Lambda_{1}\right)\right)$.

By $\phi$-symmetry and using again Lemma IV. 2

$$
\begin{aligned}
& \mu\left((g-\lambda)(r(g)-\lambda) \exp \left(\phi\left(M_{1} \cup r\left(M_{1}\right)\right)\right)\right)= \\
& \mu\left((q(g)-\lambda)(q \cdot r(g)-\lambda) \exp \left(q\left(\phi\left(M_{1} \cup r\left(M_{1}\right)\right)\right)+\phi(N)-q(\phi(N))\right)\right)
\end{aligned}
$$




$$
\begin{aligned}
&= \mu\left(( q ( g ) - \lambda ) ( p \cdot q ( g ) - \lambda ) \operatorname { e x p } \left(\phi\left(\Lambda_{1} \cup p\left(\Lambda_{1}\right)\right)+\phi\left(N \backslash\left(\Lambda_{1} \cup p\left(\Lambda_{1}\right)\right)\right)\right.\right. \\
&\left.\left.-q\left(\phi\left(N \backslash\left(M_{1} \cup r\left(M_{1}\right)\right)\right)\right)\right)\right) \\
& \leq \mu\left((q(g)-\lambda)(p \cdot q(g)-\lambda) \exp \left(\phi\left(\Lambda_{1} \cup p\left(\Lambda_{1}\right)\right)\right)\right) \\
& \times \exp \left(2 \#\left(N \backslash\left(\Lambda_{1} \cup p\left(\Lambda_{1}\right)\right)\right) \sup _{i}\left\|\phi_{i}\right\|\right) .
\end{aligned}
$$

Choosing now

$$
\lambda=\frac{\mu\left(q(g) \exp \left(\phi\left(\Lambda_{1}\right)\right)\right)}{\mu\left(\exp \left(\phi\left(\Lambda_{1}\right)\right)\right)}
$$

the right-hand side of (17) vanishes as $\mu_{\Lambda_{1} \cup \Lambda_{2}}=\mu_{\Lambda_{1}} \times \mu_{\Lambda_{2}}$. But this means by (16) that also $\mu_{M_{1} \cup M_{2}}=\mu_{M_{1}} \times \mu_{M_{2}}$.

We now arrive at the main result of this paper.

Theorem $\mathbb{I V}$. 3. Let $\phi$ be a uniformly bounded 1-body potential. The set of $\phi$-symmetric states of $\mathcal{A}_{N}$ is a simplex whose extreme points are the $\phi$-symmetric product states.

\section{Proof.}

i) Suppose that $\mu$ is a $\phi$-symmetric product state which can be decomposed as

$$
\mu=\lambda \mu_{1}+(1-\lambda) \mu_{2} \quad 0<\lambda<1
$$

and $\mu_{1}, \mu_{2} \phi$-symmetric states of $\mathcal{A}_{N}$. We have to show that $\mu_{1}=\mu_{2}=\mu$.

For any $f \in \mathcal{A}_{\Lambda}, \Lambda_{C} \mathbb{N}$ finite and for any $p \in \mathscr{Q}$ such that $\Lambda \cap p(\Lambda)=\emptyset$ it follows from Proposition III. 1 that

$$
\mu((f-\lambda)(p(f)-\lambda) \exp (\phi(\Lambda \cup p(\Lambda))))=0
$$

where

$$
\lambda=\frac{\mu(f \exp (\phi(\Lambda)))}{\mu(\exp (\phi(\Lambda)))}
$$

Therefore it follows from Lemma IV. 2 that for $i=1,2$

$$
\mu_{i}((f-\lambda)(p(f)-\lambda) \exp (\phi(\Lambda \cup p(\Lambda))))=0
$$

which means that also the $\mu_{i}$ are product states and hence coincide with $\mu$. ii) Suppose that $\mu$ is a $\phi$-symmetric state of $\mathcal{A}_{N}$ which is not a product state.

As $\sup _{i}\left\|\phi_{i}\right\|<\infty$ we can choose a strictly increasing sequence $\left\{n_{1}, n_{2}, \cdots\right\}$ in $\mathbb{N}$ such that 


$$
\phi_{\infty}=\lim _{k \rightarrow \infty} \phi_{n_{k}}(x), \quad x \in \mathcal{K}
$$

exists $\mu$ a.e.

Furthermore, we denote for $\Lambda \subset \mathbb{N}$ finite by $\phi_{\infty}(\Lambda) \in \mathcal{L}^{\infty}\left(\mathcal{K}^{N}, \mu\right)$ the function

$$
\left(x_{1}, \cdots, x_{\#(A)}\right) \rightarrow \sum_{j=1}^{\#(A)} \phi_{\infty}\left(x_{j}\right) .
$$

As $\mu$ is not a product state of $\AA_{N}$ it is possible to find finite subsets $\Lambda_{1}$ and $\Lambda_{2}$ of $N, \Lambda_{1} \cap \Lambda_{2}=\emptyset$ and $g \in \mathcal{A}_{\Lambda_{1}}$ and $f \in \mathcal{A}_{\Lambda_{2}}$ such that

$$
\mu(g f) \neq \frac{\mu\left(g \exp \left(\phi\left(\Lambda_{2}\right)-\phi_{\infty}\left(\Lambda_{2}\right)\right)\right)}{\left.\mu\left(\exp \left(\phi\left(\Lambda_{2}\right)-\phi_{\infty}\left(\Lambda_{2}\right)\right)\right)\right)} \mu(f)
$$

and one can assume without loss of generality that

$$
0<f<\exp \left(-2 \#\left(\Lambda_{2}\right) \sup _{i}\left\|\phi_{i}\right\|\right) \text {. }
$$

Define now for $r=1,2, \cdots$ states $\mu_{r}$ of $\mathcal{A}_{N}$ by :

$$
\mu_{r}(h)=\frac{\mu\left(h q_{r}(f) \exp \left(\phi\left(q_{r}\left(\Lambda_{2}\right)\right)-q_{r}\left(\phi\left(\Lambda_{2}\right)\right)\right)\right)}{\mu\left(q_{r}(f) \exp \left(\phi\left(q_{r}\left(\Lambda_{2}\right)\right)-q_{r}\left(\phi\left(\Lambda_{2}\right)\right)\right)\right)} \quad h \in \mathcal{A}_{N}
$$

where $q_{r} \in \mathscr{Q}$ is such that $q_{r}^{2}=\mathrm{id}, q_{r}\left(\Lambda_{2}\right)=\left\{n_{(r-1) \#\left(\Lambda_{2}\right)+1} ; \cdots, n_{r \#\left(\Lambda_{2}\right)}\right\}$ and $q_{r}=$ id on $N \backslash\left(\Lambda_{2} \cup q_{r}\left(\Lambda_{2}\right)\right)$. Let $\mu_{\infty}$ be a weak ${ }^{*}$-limit point of the $\mu_{r}$ and let $\left\{r_{1}, r_{2}, \cdots\right\}$ be a sequence in $N$ tending to $\infty$ such that

$$
\lim _{k} \mu_{r_{k}}=\mu_{\infty} \text {. }
$$

By construction $\mu_{\infty}$ is $\phi$-symmetric and for $h \in \mathcal{C}\left(\mathcal{K}^{N \backslash \Lambda^{2}}\right)$ one has

$$
\begin{aligned}
\mu_{\infty}( & \left.\exp \left(\phi\left(\Lambda_{2}\right)-\phi_{\infty}\left(\Lambda_{2}\right)\right)\right) \\
= & \lim _{k} \mu_{r_{k}}\left(h \exp \left(\phi\left(\Lambda_{2}\right)-\phi_{\infty}\left(\Lambda_{2}\right)\right)\right) \\
= & \lim _{k} \frac{\mu\left(h q_{r_{k}}(f) \exp \left(\phi\left(\Lambda_{2}\right)-\phi_{\infty}\left(\Lambda_{2}\right)+\phi\left(q_{r_{k}}\left(\Lambda_{2}\right)\right)-q_{r_{k}}\left(\phi\left(\Lambda_{2}\right)\right)\right)\right)}{\mu\left(q_{r_{k}}(f) \exp \left(\phi\left(q_{r_{k}}\left(\Lambda_{2}\right)\right)-q_{r_{k}}\left(\phi\left(\Lambda_{2}\right)\right)\right)\right)} \\
= & \lim _{k} \frac{\mu\left(h q_{m_{k}}(f) \exp \left(\phi\left(\Lambda_{2}\right)-q_{r_{k}}\left(\phi\left(q_{r_{k}}\left(\Lambda_{2}\right)\right)\right)+\phi\left(q_{r_{k}}\left(\Lambda_{2}\right)\right)-q_{r_{k}}\left(\phi\left(\Lambda_{2}\right)\right)\right)\right)}{\mu\left(q_{r_{k}}(f) \exp \left(\phi\left(q_{r_{k}}\left(\Lambda_{2}\right)\right)-q_{r_{k}}\left(\phi\left(\Lambda_{2}\right)\right)\right)\right)} \\
= & \frac{\mu(h f)}{\lim _{k} \mu\left(q_{r_{k}}(f) \exp \left(\phi\left(q_{r_{k}}\left(\Lambda_{2}\right)\right)-q_{r_{k}}\left(\phi\left(\Lambda_{2}\right)\right)\right)\right)}
\end{aligned}
$$

which implies that

$$
\lim _{k} \mu\left(q_{r_{k}}(f) \exp \left(\phi\left(q_{r_{k}}\left(\Lambda_{2}\right)\right)-q_{r_{k}}\left(\phi\left(\Lambda_{2}\right)\right)\right)\right)=\frac{\mu(f)}{\mu_{\infty}\left(\exp \left(\phi\left(\Lambda_{2}\right)-\phi_{\infty}\left(\Lambda_{2}\right)\right)\right)}
$$


and for $h \in \mathcal{C}\left(\mathcal{K}^{N \backslash \Lambda_{2}}\right)$

$$
\frac{\mu_{\infty}\left(h \exp \left(\phi\left(\Lambda_{2}\right)-\phi_{\infty}\left(\Lambda_{2}\right)\right)\right)}{\mu_{\infty}\left(\exp \left(\phi\left(\Lambda_{2}\right)-\phi_{\infty}\left(\Lambda_{2}\right)\right)\right)}=\frac{\mu(h f)}{\mu(f)} .
$$

Putting $h=g$ it follows from (18) that $\mu_{\infty} \neq \mu$. On the other hand by (19):

$$
\mu_{\infty} \leq \frac{\mu_{\infty}\left(\exp \left(\phi\left(\Lambda_{2}\right)-\phi_{\infty}\left(\Lambda_{2}\right)\right)\right)}{\mu(f)} \mu
$$

and therefore $\mu$ cannot be extremal $\phi$-symmetric.

The fact that the $\phi$-symmetric states are a simplex is now an immediate consequence of Proposition III. 4.

In order to conclude this section the following remark on the condition $\sup _{i}\left\|\phi_{i}\right\|<\infty$

should be made.

One can only expect Theorem IV. 3 to hold if $\phi$-symmetric product states are two by two disjoint. Moreover one would like to have such a decomposition not only for $\phi$ but for all multiples $\beta \phi, 0<\beta<\infty$, of $\phi$ which corresponds to the whold range of temperatures if one studies $\phi$-symmetric equilibrium states.

The necessary and sufficient condition on $\phi$ in order that $\beta \phi$-symmetric product states be two by two disjoint for all $0<\beta<\infty$ turns out to be : $\forall x_{1}$, $x_{2} \in \mathcal{K} \exists$ a strictly increasing sequence $\left\{n_{1}, n_{2}, \cdots\right\}$ in $\mathbb{N}$ and opens

$$
\mathcal{O}_{1}, \mathcal{O}_{2} \subset \mathcal{K} \text { with } x_{i} \in \mathcal{O}_{i}, \quad i=1,2,
$$

such that

$$
\lim _{N} \frac{1}{N \ln N} \sup _{x_{1} \in \mathcal{O}} \sum_{x_{2} \in \mathcal{O}_{2} i=1}^{N}\left|\phi_{n_{t}}\left(x_{1}\right)-\phi_{n_{t}}\left(x_{2}\right)\right|=0 .
$$

This condition essentially means that $\phi$-symmetric product states will fail to be disjoint only because some points in $\mathcal{K}$ almost don't contribute to the state because their potential energy is too high. A proof of Theorem IV. 3 similar to that in Section IV breaks down if uniform boundedness of $\phi$ is replaced by the condition (20), although some partial results indicate that the condition (20) is a good candidate to replace the uniform boundedness. 


\section{Acknowledgment}

It is a pleasure to thank Professor H. O. Georgii for pointing out ref. [14] to me.

\section{References}

[1] De Finetti, B., La prévision: ses lois logiques, ses sources subjectives, Ann. Inst. H. Poincaré, 1 (1937), 1-68.

[2] Hewitt E. and Savage, L. S., Symmetric measures on Cartesian products, Trans. Am. Math. Soc., 80 (1955), 470-501.

[ 3 ] St $\varnothing$ rmer, E., Symmetric states of infinite tensor products of $C^{*}$-algebras, J. Funct. Anal., 3 (1969), 48-68.

[ 4 ] Hudson R. L. and Moody, G. R., Locally normal symmetric states and an analog of de Finetti's theorem, Z. Wahrscheinlichkeitstheorie verw. Gebiete. 33 (1976), 343-351.

[5] Thirring, W., On the mathematical structure of the B.C.S. model II, Commun. Math. Phys., 7 (1968), 181.

[ 6 ] Dicke. Rotto, Coherence in spontaneous radiation processes, Phys. Rev., 93 (1954), 99-110.

[ 7] Fannes M. and Verbeure, A., Correlation inequalities and equilibrium states, Commun. Math. Phys. 55 (1977), 125-131 and Correlation inequalities and equilibrium states II, Commun. Math. Phys., 57 (1977), 165-171.

[ 8 ] Fannes, M. Spohn H. and Verbeure, A., Equilibrium states for mean field models, J. Math. Phys., 21 (1980), 355-358.

[ 9 ] Huang, K. Yang C.N. and Luttinger, J. M., Imperfect Bose gas with hard-sphere interactions, Phys. Rev., 105 (1957), 776-784.

[10] Jelinek, F. B.C.S. spin model, its thermodynamic representations and automorphisms, Commun. Math. Phys., 9 (1968), 169.

[11] Van den Berg, M. Lewis J. T. and Pulè, J. V., The large deviation principle and some models of an interacting Boson gas, preprint Dublin Institute for Advanced Studies.

[12] Lanford III O. E. and Ruelle, D., Observables at infinity and states with short range correlations in statistical mechanics, Commun. Math. Phys., 13 (1969), 194.

[13] Sullivan, W. G., Mean square relaxation times for evolutions of random fields, Commun. Math. Phys., 40 (1975), 249.

[14] Kakutani, S., On the equivalence of infinite product measures, Ann. Math., 49 (1948), 214. 